\title{
L istado taxonómico y distribución de los tricópteros inmaduros del departamento del Tolima
}

\author{
E dwin 0. López-Delgado1,*, J esús M. Vásquez-R amos ${ }^{1,2}$, G ladys R einoso-F lórez ${ }^{1,3}$ \\ ${ }^{1}$ Grupo de Investigación en Zoología, Facultad de Ciencias, Universidad del Tolima, Ibagué, Colombia \\ ${ }^{2}$ Programa de Biología, Universidad de los Llanos, Villavicencio, Colombia \\ ${ }^{3}$ Programa de Biología, Universidad del Tolima, Ibagué, Colombia
}

\begin{abstract}
Resumen
El presente listado taxonómico y de distribución de tricópteros inmaduros corresponde al material biológico depositado en la Colección Zoológica de la Universidad del Tolima- CZUT-Ma. Se registraron 14 de las 15 familias y 35 de los 45 géneros reportados para Colombia. Las familias más abundantes fueron Hydropsychidae, Hydroptilidae, Leptoceridae, y los géneros, Smicridea, Leptonema y Hydroptila. Con relación a la distribución altitudinal, los géneros con los mayores rangos de distribución fueron Neotrichia, Leucotrichia y Smicridea, recolectados desde los $244 \mathrm{~m}$ hasta los $3.533 \mathrm{~m}$. Con el fin de establecer las relaciones entre los géneros de Trichoptera y las zonas de vida, se hizo un análisis de correspondencia cuyos resultados permitieron identificar que la mayor riqueza específica de este grupo faunístico en el departamento del Tolima se concentró en las zonas de vida correspondientes a bosque seco tropical, bosque húmedo premontano y bosque húmedo montano bajo.
\end{abstract}

Palabras clave: Trichoptera, Tolima, zonas de vida, correspondencia, CZUT.

Taxonomic list and distribution of immature caddisflies in the Department of Tolima

\begin{abstract}
A bstract
This taxonomic list of the Trichoptera larvae references data from the Zoological Voucher Collection at Universidad del Tolima, Colombia (Colección Zoológica de la Universidad del Tolima - CZUT-Ma). This collection includes 14 families and 35 genera of Trichoptera (out of the 15 families and 45 genera currently registered in Colombia). The most abundant families were Hydropsychidae, Hydroptilidae and Leptoceridae. The most abundant genera were Smicridea, Leptonema and Hydroptila. Regarding altitudinal distribution of the taxa found in the collection, Neotrichia, Leucotrichia and Smicridea exhibited the widest distribution, since they were found from $244 \mathrm{~m}$ to 3533m. Results from a correspondence analysis performed on the taxa vs Holdridge life zones data, revealed that the highest species richness of the order Trichoptera in Tolima (Colombia) concentrated in the tropical dry forest, wet pre-mountain forest and lower wet mountain forest zones.
\end{abstract}

K ey words: Trichoptera, Tolima, Holdridge life zones, correspondence analysis, CZUT.

\section{Introducción}

Los tricópteros son organismos holometábolos, pues desarrollan los estados de huevo, larva, pupa y adulto (Posada \& R oldán, 2003). Sus formas inmaduras son acuáticas y los adultos son voladores. Las larvas se encuentran en diferentes hábitats, principalmente en ecosistemas lóticos, presentan generalmente cinco estadios larvales con tamaños entre 1 y $30 \mathrm{~mm}$, y pueden ser libres o vivir en refugios que construyen con seda y diferentes materiales orgánicos e inorgánicos. Estos organismos presentan tres regiones corporales bien desarrolladas y diferenciadas: cabeza, tórax y abdomen. La cabeza está esclerotizada, los ojos son pequeños y simples, presenta piezas bucales masticadoras bien desarrolladas y con una glándula productora de seda ( $M$ uñoz-Q uesada, 2004). El tórax posee tres segmentos bien diferenciados, el protórax siempre aparece esclerotizado y los restantes tienen diversos grados de esclerotización, proyectándose en cada uno de ellos un par de patas bien desarrolladas. El abdomen está dividido en nueve segmentos, que pueden o no tener branquias o pequeñas regiones esclerotizadas; en el último segmento abdominal se encuentran un par de propatas anales, cada una de ellas con una uña terminal, que pueden variar de forma en las distintas especies (W iggins, 1996; Angrisano \& Sganga, 2009).

En Colombia los tricópteros se distribuyen ampliamente en la región suroccidental, especialmente en las vertientes orientales y occidentales de las cordilleras Oriental y

\footnotetext{
*C orrespondencia:

Edwin Orlando López Delgado, eolopezd@gmail.com

Recibido: 2 de septiembre de 2014

A ceptado: 13 de marzo de 2015
} 
Occidental (Quintero \& R ojas, 1987). Se han registrado un total de 15 familias, 45 géneros y 210 especies válidas, de cuyos organismos solo se ha reportado el $10 \%$ (M uñoz-Q uesada, 2004, Posada \& R oldán, 2003). En el departamento del Tolima se han registrado 30 géneros de larvas de tricópteros distribuidos en 13 familias presentes en las cuencas de los ríos Coello, Prado, Amoyá y Totare (Vergara, et al., 1994; R einoso, 1998, 2001; C arrillo, 2002; G uevara, 2004; G uevara, et al., 2006; Vásquez, et al., 2006, 2007, 2008, 2008a, 2008b; López, et al., 2006; López, 2007, R amírez, et al., 2007, 2008a, 2008b, R einoso, et al., 2007; Vásquez \& Ramírez, 2008, López \& R einoso, 2008).

Ecológicamente, los tricópteros juegan un papel muy importante en los ecosistemas acuáticos, ya que hacen parte de la cadena trófica y son el principal alimento de una gran variedad de peces, aves y otros vertebrados que se encuentran cerca de los cuerpos de agua (W iggins, 1996; Domínguez \& Fernández, 2009). Además, se han posicionado como grupo potencialmente útil en los estudios sobre calidad del agua debido a su sensibilidad frente a los cambios físicos y químicos (G uevara, et al., 2006; Valero, et al., 2001). Por lo tanto, surge la necesidad de desarrollar estudios encaminados a conocer la riqueza taxonómica de este grupo y su distribución en las diferentes zonas de vida en la cordillera Central de los Andes colombianos.

\section{Materiales y métodos}

El listado de géneros de los tricópteros inmaduros se compiló a partir de la información de los organismos depositados en la Colección Zoológica de la Universidad del Tolima (CZUT-Ma). Los ejemplares provienen principalmente de los estudios de biodiversidad regional que se han desarrollado en el marco del convenio entre la Universidad del Tolima y la Corporación Autónoma Regional del Tolima CORTOLIMA, como parte del Plan de Ordenamiento de las Cuencas Hidrográficas de Coello, Prado, Totare, Lagunillas, Anchique y Saldaña (subcuencas Amoyá, Anamichú, Patá, Hereje) del departamento de Tolima, Colombia.

A partir de los datos obtenidos de la colección biológica se elaboraron matrices de abundancia por familias y géneros con sus respectivas localidades, altitudes, coordenadas geográficas y zonas de vida, de acuerdo a la clasificación de Holdridge (1987) y con la ayuda del programa Microsoft Excel 2010. Además, con este mismo programa se calcularon y graficaron los datos sobre abundancia relativa. Los datos de distribución altitudinal se calcularon restando del valor más alto de altitud el menor registrado para cada uno de los taxones.

Con el fin de determinar la distribución de los géneros con respecto a las zonas de vida se hizo un análisis de correspondencia, en el cual se relacionaron los géneros con las zonas de vida registradas en cada punto de muestreo. Este análisis de ordenación se hizo con el programa estadístico CANOCO 4.5 (L epš, J ., \& Šmilauer, P., 2003).

\section{Resultados}

Se presenta a continuación el listado taxonómico de los géneros de los tricópteros inmaduros registrados en el departamento del Tolima en las cuencas de los ríos Coello, Prado, Totare, Lagunillas, Anchique y Saldaña (subcuencas Amoyá, Anamichú, Patá, Hereje) (Tabla 1S, http://www.raccefyn.co/ index.php/raccefyn/article/downloadSuppFile/108/691 y Tabla 2S, http://www.raccefyn.co/index.php/raccefyn/ article/downloadSuppFile/108/692), en el que se reportan 35 géneros distribuidos en 14 familias. Las familias más abundantes fueron Hydropsychidae (42,7 \%), Hydroptilidae (18,16 \%) y Leptoceridae (9,26 \%), y las menos abundantes y poco frecuentes, Anomalopsychidae (0,34 \%), Ecnomidae $(0,18 \%)$ y Xiphocentronidae (0,07 \%) (Figura 1$)$.

Con relación a los géneros, los más abundantes fueron Smicridea (24,99 \%), Leptonema (17,13 \%) e Hydroptila (9,62 \%), los cuales representan el 51,74\% del total de organismos registrados para el departamento del Tolima. En contraste, los menos abundantes fueron Macronema (0,03 \%), Mortoniella y Macrostemum (ambos con 0,02 \%) y, por último, Mexitrichia (0,01 \%) (Figura 2).

\section{Distribución altitudinal}

En cuanto a la distribución altitudinal, los géneros que presentaron los rangos más amplios fueron Neotrichia, Leucotrichia y Smicridea, mientras que los rangos más bajos fueron registrados por Ithytrichia, Banyallarga $\mathrm{y}$ Anomalocosmoecus. Es importante destacar que los géneros Mexitrichia y Macrostemum solo se registraron en una de las localidades de estudio, por lo tanto su valor en la distribución altitudinal fue puntual (Figura 3).

\section{Distribución de los taxones de acuerdo a las zonas de vida}

Con relación a las zonas de vida, los géneros Smicridea, Atopsyche, Helicopsyche y Nectopsyche se distribuyeron ampliamente en la mayoría de las zonas del departamento, desde la de bosque seco tropical (bs-T) hasta la de bosque pluvial subalpino (bp-SA). En contraste, los taxones

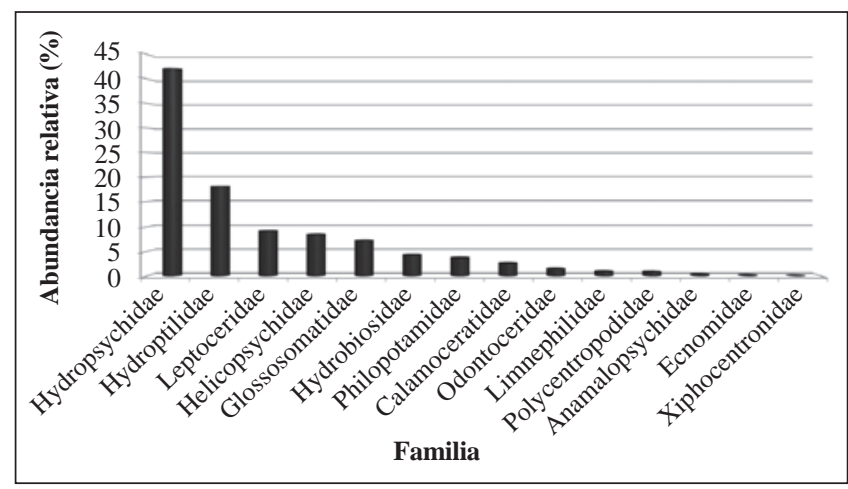

Figura 1. Abundancia relativa de las familias de las larvas del orden Trichoptera recolectadas en el departamento del Tolima entre el 2004 y el 2008 


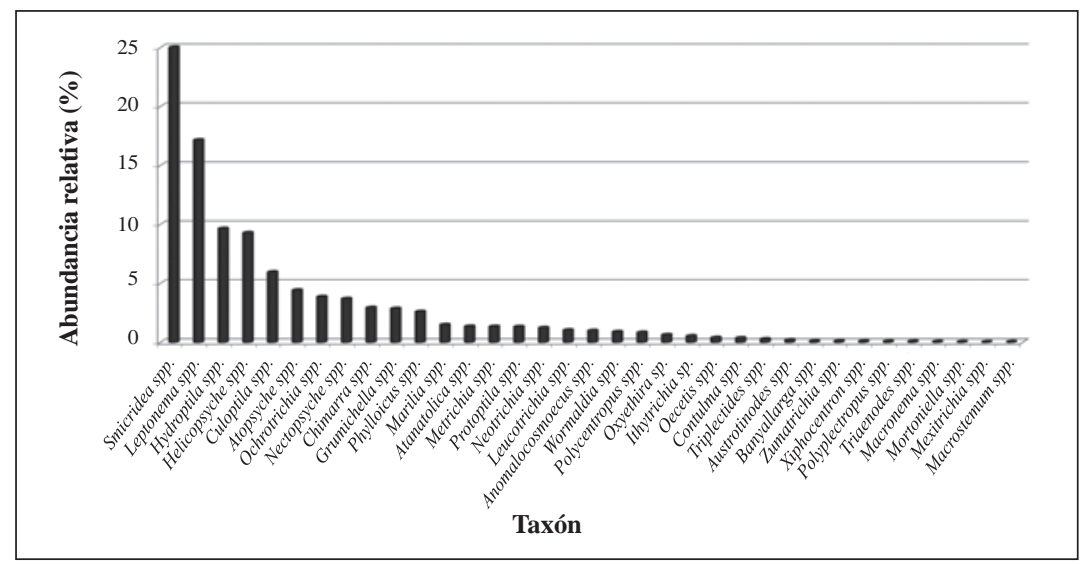

Figura 2. Abundancia relativa de los géneros de las larvas del orden Trichoptera recolectadas en el departamento del Tolima entre el 2004 y el 2008

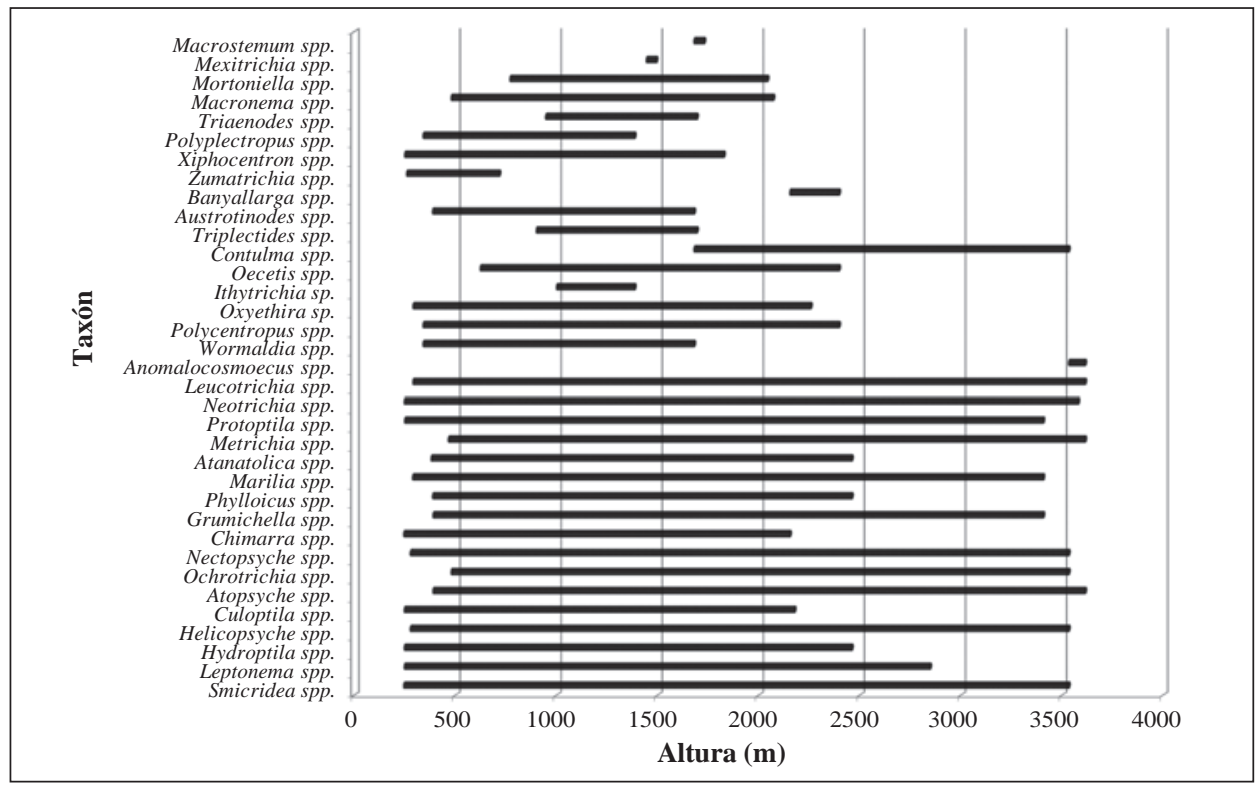

Figura 3. Distribución altitudinal de los géneros de las larvas del orden Trichoptera recolectadas en el departamento del Tolima

Anomalocosmoecus, de la zona de bosque pluvial subalpino (bp-SA); Banyallarga, de la de bosque húmedo montano bajo (bh-MB); Macrostemum, de la de bosque húmedo montano bajo (bh-MB), y Mexitrichia, de la de bosque húmedo premontano (bh $\mathrm{PM}$ ), se registraron en una sola zona de vida; sin embargo, es importante tener en cuenta que el registro de estos dos últimos taxones es puntual, pues solo se han recolectado en una localidad de las 157 evaluadas (Tabla 1S) (Figura 4).

\section{Discusión}

Para Colombia se han reportado 15 familias y 45 géneros de Trichoptera (M uñoz-Q uesada, 2004, Posada \& R oldán, 2003). Catorce de estas familias y 35 de los géneros se han registrado en el departamento del Tolima, lo que evidencia la gran representatividad de la fauna de tricópteros en este departamento, la cual probablemente aumentará a medida que se sigan fomentando y haciendo estudios taxonómicos y ecológicos en la región.

Según los datos obtenidos de la Colección Zoológica de la Universidad del Tolima, la familia más abundante en las diferentes cuencas evaluadas fue Hydropsychidae. Estos resultados concuerdan con los reportados en diferentes estudios hechos en el Tolima y en otras regiones del país, en los cuales se ha identificado que los organismos pertenecientes a esta familia se caracterizan por su versatilidad y capacidad para colonizar diferentes tipos de sustratos como rocas, arena, grava, cieno y hojarasca (G uevara, 2004; M uñoz-Q, 2004; López, et al., 2006; López, 2007; Vásquez-R amos, 2008). Además, se ha evidenciado que la mayoría de individuos de esta familia, especialmente de los géneros Smicridea y 


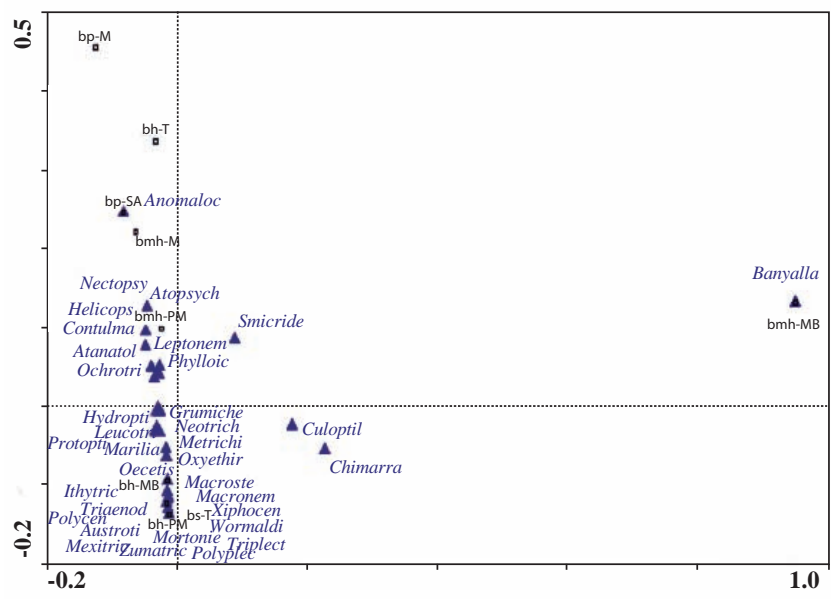

Figura 4. Distribución de los géneros de las larvas del orden Trichoptera recolectadas en el departamento del Tolima entre el 2004 y el 2008 en las diferentes zonas de vida evaluadas

Leptonema, poseen una gran capacidad para tolerar diferentes tipos de ambientes, desde aguas completamente limpias hasta aguas con algún grado de intervención antrópica (C orrea, et al., 1981; Q uintero \& R ojas, 1987; Vergara, et al., 1994; Roldán, 1996; G uevara, 2004; M uñoz-Q, 2004; López, et al., 2006; M osquera, et al., 2006; López, 2007; Vásquez-R amos, 2008; V ásquez-R amos, et al., 2010).

Otra de las familias más abundantes fue la Hydroptilidae, la cual se caracteriza por ser una de las más diversas del orden y por habitar zonas de corrientes tanto rápidas como lentas, principalmente en zonas tropicales (M oor \& I vanov, 2007). Igualmente, esta familia se ha encontrado en todas las regiones del mundo y en todos los pisos altitudinales colonizando diferentes tipos de sustratos como vegetación acuática, musgos y zonas de salpicaduras de sustratos pedregosos donde se alimenta del perifiton que crece sobre estas superficies (Flint, 1991; Posada \& Roldán, 2003; M uñoz-Q, 2004; M oor \& Ivanov, 2007; Vásquez-Ramos, 2008; O liveira, et al., 2005; G ómez-A guirre, et al., 2009; Springer, 2010).

Así mismo, la familia Leptoceridae registró una abundancia considerable, siendo también una de las más diversas del orden. Esta familia presenta diversos comportamientos tróficos, como en las larvas detritívoras o predadoras, en tanto que algunas se alimentan del perifiton, especialmente de diatomeas que crecen sobre las rocas de las zonas de salpicaduras (M uñoz-Q, 2004). Algunos autores como Holzenthal (1994) consideran que este grupo de organismos se encuentra en aguas con corrientes rápidas o lentas y poseen ventajas adaptativas como la construcción de refugios en forma cónica que les brindan protección y los hacen poco llamativos para sus depredadores.

Las familias Anomalopsychidae, Ecnomidae y Xiphocentronidae registraron los valores más bajos de abundancia en las cuencas evaluadas, por lo que se podría considerar a estas tres familias como raras y poco frecuentes en el departamento del Tolima, lo cual coincide con lo reportado en otros departamentos por Posada \& Roldán (2003), M uñoz-Q, 2004 y Ballesteros, et al., 1997. Dichos hallazgos podrían indicar que estos organismos poseen una alta sensibilidad a las variaciones ambientales y que presentan cierto grado de selectividad de microhábitats específicos.

Los individuos del género Contulma (Anomalopsychidae) se registraron en aguas con corrientes rápidas y sustratos con grava y arena desde los 1.680 m hasta los 3.533 m. Así mismo, los organismos registrados de la familia Ecnomidae, pertenecientes al género Austrotinodes, se recolectaron en corrientes rápidas con sustratos de roca, arena y hojarasca, desde los 387 m hasta los 1.680 m. Wiggins (2004) considera que los organismos de estas familias son muy raros y poco frecuentes en las recolecciones, por lo que algunos aspectos de su ecología y su biología aún son objeto de estudio. Por último, la familia Xiphocentronidae, registrada desde los 249 hasta los $1.827 \mathrm{~m}$, se caracteriza por construir elaborados tubos de seda y arena pegados a las paredes de las piedras (Schmid, 1982); los organismos pertenecientes a esta familia se encontraron en corrientes de montaña densamente cubiertas por bosque, lo que podría indicar su preferencia por colonizar sustratos específicos, especialmente zonas de abundante vegetación riparia.

Los géneros más abundantes fueron Leptonema y smicridea, pertenecientes a la familia Hydropsychidae, los cuales se caracterizan por colonizar una amplia gama de sustratos (roca, hojarasca, grava, etc.), y construir extensas redes de seda que les facilitan la captura del alimento, convirtiéndolos en uno de los grupos más abundantes dentro del orden (Wiggins, 2004); algunos autores consideran que los taxones de la familia Hydropsychidae abarcan alrededor de $80 \%$ de todos los tricópteros en el continente americano (Davis, et al., 1991; Quintero \& Rojas, 1987; Vergara, et al., 1994; Roldán, 1996; G uevara, 2004; M uñoz-Q, 2004; M osquera, et al., 2006; López, 2007; Vásquez-R amos, 2008; Angrisano \& Sganga, 2009; Vásquez-Ramos, et al., 2010 ), lo cual se ve reflejado en la alta densidad de organismos pertenecientes a estos taxones registrada en todas las regiones del país.

El género Hydroptila, perteneciente a la familia Hydroptilidae, fue el siguiente en orden de abundancia, situación que se debe, en parte, a su pequeño tamaño (1-5 mm), lo que le permite alcanzar densidades considerables en áreas pequeñas, especialmente en los tallos y hojas de plantas acuáticas (Flint, 1991).

Dos de los géneros menos abundantes en las diferentes cuencas evaluadas fueron Macronema y Macrostemum, pertenecientes a la familia Hydropsychidae. Estos taxones se caracterizan por ser raros y poco frecuentes en las recolecciones y, al parecer, su distribución está limitada por sus hábitos alimenticios, ya que construyen redes con 
diámetros muy pequeños que les permiten alimentarse del perifiton más fino alojado en la columna de agua; además, los microhábitats que ocupan son difíciles de evaluar con los métodos de recolección convencionales, ya que se encuentran entre las grietas de las rocas y otros lugares de difícil acceso (Wallace, 1976; Wiggins, 1996).

\section{Distribución altitudinal y zonas de vida}

El amplio gradiente altitudinal presentado por los géneros Neotrichia, Leucotrichia y Smicridea se ha reportado en diferentes estudios llevados a cabo en la región andina (Ballesteros, et al., 1997; Posada \& R oldán, 2003; G uevara, 2004; López, et al., 2006; M osquera, et al., 2006; López, 2007; Vásquez-R amos, 2008). Algunos investigadores como Wiggins (1996), mencionan que estos organismos presentan una amplia distribución en el Neotrópico y que suelen ser euritolerantes, pues pueden encontrarse en ríos y quebradas con diferentes tipos de corrientes y temperaturas. Otro posible factor que puede influir en la amplia distribución de estos organismos es la capacidad que tienen los adultos para volar, ya que los individuos de estas dos familias poseen alas fuertes que les permiten desplazarse largas distancias y colonizar diferentes lugares de los cuerpos de agua (Davis, et al., 1991).

Por otra parte, los taxones Ithytrichia, Banyallarga y Anomalocosmoecus, presentaron una variación altitudinal muy baja, resultado que podría indicar que estos géneros son sensibles a las variaciones de factores físicos como la temperatura, la materia orgánica disponible o el oxígeno disuelto, o a los cambios en la concentración química de nitritos, fosfatos, y en la conductividad eléctrica, entre otros (López, 2007; Vásquez-R amos, 2008).

En cuanto a la distribución altitudinal, la precipitación y la temperatura, la mayor riqueza de la fauna tricóptera se concentró en las zonas de vida de bosque seco tropical bs$\mathrm{T}$, de bosque húmedo premontanao y de bosque húmedo montano bajo, hecho probablemente está relacionado con la gran heterogeneidad y diversidad de hábitats que se encuentran en los cuerpos de agua de estas zonas, así como con una mayor cobertura riparia, la cual juega un papel importante en la distribución y el aporte de energía a las cadenas tróficas de los ecosistemas acuáticos (Vásquez, 2012). Los géneros que se distribuyeron ampliamente en las diferentes zonas de vida correspondieron a Smicridea, Atopsyche, Helicopsyche y Nectopsyche.

Por otra parte, los taxones Macrostemum (bh-MB), Mexitrichia (bh-PM), Banyallarga (bh-MB) y Anomalocosmoecus (bpSA), se registraron solamente en una zona de vida. Estos individuos al parecer presentan una distribución puntual con relación a la temperatura, la precipitación y la altitud, las cuales posiblemente influyen en su desarrollo óptimo (G uevara, 2004; López, et al., 2006; Vásquez-Ramos \& R amírez, 2008). El género Anomalocosmoecus, por ejemplo, se registró solamente en la zona de vida de bosque pluvial subalpino por encima de los $3.000 \mathrm{~m}$, con aguas frías muy oxigenadas y con poca o nula intervención antrópica (L ópez, et al., 2006; G uevara, 2004), lo que podría ser indicativo de la selectividad de estos organismos por condiciones ambientales específicas y de su limitada distribución en el departamento del Tolima.

Es importante tener en cuenta que la distribución del orden Trichoptera obedece a un sinnúmero de variables físicas, químicas y ecológicas. Entre ellas vale la pena mencionar la oferta alimenticia, que sin lugar a dudas es un factor limitante para la distribución, la colonización y la diversificación del orden (B onada, et al., 2005). Debe resaltarse el hecho de que la mayoría de los ecosistemas altoandinos se caracterizan principalmente por aguas pobres en nutrientes (oligotróficas), en las cuales la oferta de algas, especialmente clorofíceas y cianofíceas, es muy baja (Ramírez \& Viña, 1998), lo cual se evidencia en la poca abundancia y riqueza de la fauna tricóptera en las zonas de vida ubicadas en alturas superiores a los $2.900 \mathrm{~m}$ en el departamento del Tolima.

Estos resultados constituyen un aporte valioso por la información taxonómica que aportan sobre la distribución y las zonas de vida en las que se encuentran los géneros de tricópteros inmaduros en ambos flancos de la cordillera Central, y en el flanco occidental de la cordillera Oriental de la zona central de los Andes colombianos. Estos resultados son parte esencial del eje estructural para los futuros planes de manejo y conservación de las cuencas del departamento, especialmente en lo referente al uso responsable y sostenible del recurso hídrico.

\section{Conclusiones}

El departamento del Tolima alberga el $93 \%$ de las familias y el $78 \%$ de los géneros de tricópteros reportados para Colombia, de los cuales un alto porcentaje habita en las zonas de vida de bosque seco tropical, bosque húmedo premontano y bosque húmedo montano bajo.

Esta revisión permitió establecer que en el departamento del Tolima la familia Hydropsychidae, representada por los géneros Leptonema y Smicridea, fue la más abundante y frecuente, así como la de mayor distribución, corroborando así los resultados obtenidos en otros departamentos y evidenciando la alta capacidad de estos organismos para tolerar ambientes diversos.

Los taxones Anomalopsychidae (Contulma), Ecnomidae (Austrotinodes) y Xiphocentronidae (Xyphocentron) fueron los menos abundantes, lo que sugiere que estos organismos son raros y poco frecuentes en el departamento.

\section{Agradecimientos}

Agradecemos a la Colección Zoológica de la Universidad del Tolima (CZUT) por facilitar la información necesaria para la elaboración del presente documento. 


\section{Información suplementaria}

Tabla 1S. Listado de los géneros de tricópteros inmaduros registrados en el departamento del Tolima
Tabla 2S. Listado de las estaciones donde se han registrado larvas del orden Trichoptera en el departamento del Tolima

\section{Conflicto de intereses}

Los autores declaran no tener conflicto de intereses.

\section{R eferencias}

Angrisano, E. B. (1995). Insecta Trichoptera. En: Lopretto, E. y Tell, G., (Eds.). Ecosistemas de aguas continentales: metodologías para su estudio. La Plata, Argentina: Ediciones Sur, Tomo III.

Angrisano, E. B. \& Sganga, J . V. (2009). Trichoptera. En: E. Domínguez \& H. R. Fernández (Eds.). Macroinvertebrados bentónicos sudamericanos. Primera edición. Tucumán: Fundación Miguel Lillo. p. 255-308.

Ballesteros, Y. V., Zúñiga, M. C ., R ojas de Hernández, A. M. (1997). Distribution and structure of the order Trichoptera in various drainages of the Cauca River basin, Colombia, and their relationship to water quality. En: Proceedings of the 8th International Symposium on Trichoptera. Ohio: Ohio Biological Survey. p. 19-23.

Lepš, J „, \& Šmilauer, P. (2003). Multivariate analysis of ecological data using CANOCO. United States of America. Cambridge university press.

Bonada, N., Zamora-M uñoz, C., Rieradevall, M, Prat, N. (2005). Ecological and historical filters constraining spatial caddisfly distribution in Mediterranean rivers. Freshwater Biology. 50: 781-797.

Carrillo D. (2002). Aspectos bioecológicos de los macroinvertebrados en el embalse de Hidroprado, departamento del Tolima. Trabajo de grado (Biólogo). Universidad del Tolima, Facultad de Ciencias Básicas, Departamento de Biología, Ibagué.

Correa M., Machado, T., Roldán, G . (1981) Taxonomía y ecología del orden Trichoptera en el departamento de Antioquia en diferentes pisos altitudinales. Actualidades Biológicas. 10 (36): $35-48$

Davis, B., Hudson, P., A rmitage, B. (1991). Distribution and abundance of caddisflies (Trichoptera) in the ST. ClairDetroit river system. Internat. Assoc. Great Lakes. 17 (4): 522-535.

Domínguez, E. \& Fernández, H. R. 2009. Macroinvertebrados bentónicos sudamericanos. Sistemática y biología. E. Domínguez \& H. R. Fernández (Eds.). Primera edición. Tucumán: Fundanción Miguel Lillo. p. 656.

Flint, O. S. 1991. Studies of Neotropical Caddisflies, XLV: The taxonomy, phenology, and faunistics of the trichoptera of Antioquia, Colombia. Washington, D.C. Smithsonian Institution Press. Smithsonian contributions to zoology. 520: 113.
Gómez-Aguirre, A. M., Longo-Sánchez, M. C., Blanco, J. F. (2009). Macroinvertebrate assemblages in Gorgona Island streams: Spatial patterns during two contrasting hydrologic periods. Actual Biol, 31 (91): 161-178. Fecha de consulta: mayo de 2014. Disponible en: http://matematicas.udea. edu.co/ actubiol/publicaciones_pdf/2009/2 RAB 31(91), PDFs/Mss 31(91), PDFs/Ensamblaje de macroinvertebrados de las quebradas. pdf.

Guevara G . 2004. Análisis faunístico del orden Trichoptera en su estado larval en la cuenca del río Coello, departamento del Tolima. Trabajo de grado (Maestría). Universidad del Tolima, Facultad de Ciencias Básicas, Departamento de Biología, Ibagué.

G uevara, G ., López, E ., Reinoso, G ., Villa-Navarro, F. (2006). Structure and distribution of the Trichoptera fauna in a Colombian Andean river basin (Prado, Tolima) and their relationship to water quality. Proccedings of the XIIth International Symposium on Trichoptera. Mexico, D.F.

G uevara, G ., R einoso, G ., Villa-Navarro, F. (2006). Caddisfly larvae (Insecta: Trichoptera) of the Coello River basin in Tolima (Colombia): Spatial and temporal patterns and bioecological aspects. Proccedings of the XIIth International Symposium on Trichoptera. Mexico, D.F.

H oldridge, L . (1987). Ecología basada en zonas de vida. San José, Costa Rica: IICA. p. 216.

Holzenthal, R. 1994. Orden Trichoptera. En: Solís, A. (Ed.). Las familias de insectos de Costa Rica. Derechos Reservados, Instituto Nacional de Biodiversidad, Costa Rica. Santo Domingo de Heredia, Heredia, Costa Rica. Fecha de consulta: mayo 2014. Disponible en: http://www.inbio. ac.cr/papers/insectoscr/Texto83.html.

López E ., R einoso, G ., G uevara, G ., Villa, F. (2006). Estructura, distribución y relaciones con el Índice de Calidad de Aguas de la tricopterofauna en la cuenca del río Prado (Tolima, Colombia). En: F. Villa, C. Rivera, G. Reinoso, M. Núñez (Eds.). Resúmenes del VII Seminario Colombiano de Limnología y I Reunión Internacional sobre Ríos y Humedales Neotropicales. Asociación Colombiana de Limnología, Ibagué. p. 18.

López E. (2007). Análisis faunístico de las larvas del orden Trichoptera en la cuenca del río Prado y la subcuenca de Amoyá (Tolima-Colombia). Trabajo de grado (Biólogo). Universidad del Tolima, Facultad de Ciencias Básicas, Departamento de Biología, Ibagué.

López E., Reinoso, G . (2008). Variación temporal de la fauna del orden Trichoptera en la cuenca el río Prado. En: P. Landázuri (Ed.). Memorias XLIII Congreso Nacional de Ciencias Biológicas. Armenia, Quindío. p. 34. 
M oor, F.C. \& I vanov, V.D. (2008). Global diversity of caddisflies (Trichoptera: Insecta) in fresh water. Hydrobiologia. 595: 393-407.

M osquera Z., Bejarano, D., Asprilla, S. (2006). Estudio del orden Trichoptera (Insecta) en dos ecosistemas lóticos del municipio de Quibdó, Chocó-Colombia. En: F. Villa, C. Rivera, G. Flórez, M. Núñez, \& X. Carranza (Eds.). Memorias VII Seminario Colombiano de Limnología y I Reunión Internacional sobre Ríos y Humedales Neotropicales. Ibagué: León Gráficas. p. 85-91.

Muñoz-Q, F. y Paprocki, H. (2003). Claves para la identificación de las larvas de las familias neotropicales de trichoptera. Caldasia. 25 (1): 169-192.

Muñoz-Quesada, F. (2004). El orden Trichoptera (Insecta) en Colombia, II: inmaduros y adultos, consideraciones generales. En: Fernández, F, MG, Andrade \& G, Amat (Eds.). Insectos de Colombia, Volumen III. Universidad Nacional de Colombia, Bogotá D.C., p. 319-349.

Oliveira, A. M., Hamada, N., Nessimian, J . L . (2005). Chaves de identificação de larvas para famílias e gêneros de Trichoptera (Insecta) da Amazônia central, Brasil. Revista Brasileira de Entomologia. 49 (2): 181-204.

Posada J A . \& R oldán, G . (2003). Clave ilustrada y diversidad de las larvas de Trichoptera en el noroccidente de Colombia. Caldasia. 25 (1): 169-192.

Quintero, A. \& Rojas, A. (1987). Aspectos bioecológicos del orden Trichoptera y su relación con la calidad del agua. Revista Colombiana de Entomología. 13 (1): 26-38.

Ramírez F., Vásquez, J., Reinoso, G., Guevara, G. (2007). En busca de lo desconocido: una nueva familia en el bentos tolimense (Trichoptera: Xiphocentronidae). En: G. Cervantes (Ed.). Memorias del XLII Congreso Nacional de Ciencias Biológicas. Departamento de Publicaciones, Universidad Simón Bolívar, Barranquilla. p. 30.

Ramírez F., Vásquez, J ., Reinoso, G., Guevara, G . (2008a). Leptocéridos (Trichoptera) de la cuenca del río Totare, Tolima-Colombia. En: E. Peña, J. Cantera, M. Zúñiga, S. Duque, E. Londoño, L. Aguirre, B. Albán, P. Bedoya (Eds.). Resúmenes del VIII Seminario Colombiano de Limnología y I Reunión Internacional de Cuencas Bajas y Zonas Estuarinas. Universidad Autónoma de Occidente, Santiago de Cali, p. 53.

Ramírez F., Vásquez, J ., Reinoso, G ., G uevara, G . (2008b). Los tricópteros y su papel bioindicador: caso del río Totare. En: P. Landázuri (Ed.). Memorias XLIII Congreso Nacional de Ciencias Biológicas. Armenia, Quindío. p. 33

Reinoso G. (1998). Efemerópteros (Arthropoda: Insecta) del río Combeima en el trayecto comprendido entre Juntas y El Totumo municipio de Ibagué departamento del Tolima. Trabajo de grado (Maestría). Universidad de los Andes, Departamento de Ciencias Biológicas, Bogotá.

Reinoso G . (2001). Estudio bioecológico de los tricópteros del río Alvarado en el trayecto comprendido entre El Salado y Alvarado, Tolima. Universidad del Tolima, Facultad de Ciencias, Departamento de Biología.
Reinoso G., Villa, F., Esquivel, H, García, J., Vejarano, M. (2007). Biodiversidad faunística y florística de la cuenca del río Totare - Biodiversidad regional fase III. Informe final. Universidad del Tolima. Ibagué.

R einoso G., G uevara, G., Vejarano, M., G arcía, J ., Villa, F. (2008). Áreas de interés para la conservación en la cuenca del río Prado (Tolima, Colombia): macroinvertebrados y calidad de agua como factores clave. Revista de la Asociación Colombiana de Ciencias Biológicas. 20: 102-116.

Roldán, P. G . (1996). Guía para el estudio de los macroinvertebrados acuáticos del departamento de Antioquia. Bogotá (Colombia): Fondo FEN Colombia.

Roldán, P. G. (2003). Bioindicación de la calidad de agua en Colombia: uso del método BMWP/Col. Medellín (Colombia): Universidad de Antioquia.

Schmid, F. 1982. La famille des Xiphocentronides (Trichoptera: Annulipalpia). Mémoires de la Société Entomologique du Canada. 121: 1-127.

Strum, H. 1960. Die terrestrischen Puppengehäuse von Xiphocentron strumi Ross (Xiphocentronidae, Trichoptera). Zool. Jahrb. Syst. 87 (4/5): 387-394.

Valero, L., Durán, P., Arellano, E. (2001). Trichoptera como indicadora de calidad de agua. Río Albarregas. Mérida, Venezuela. Rev. Ecol. Lat. Am. 8 (1): 11-16.

Vásquez J „, Ramírez, F., R einoso, G ., G uevara, G . (2006). Preferencias de sustrato del orden Trichoptera (clase Insecta) en la cuenca del río Combeima, Ibagué - Colombia. En: F. Villa, C. Rivera, G. Reinoso \& M. Núñez (Eds.). Resúmenes del VII Seminario Colombiano de Limnología y I Reunión Internacional sobre Ríos y Humedales Neotropicales. Asociación Colombiana de Limnología, Ibagué. p. 138.

Vásquez J ., Ramírez, F., Reinoso, G., Guevara, G. (2007). Aspectos bioecológicos del orden Trichoptera en su estado larval en la cuenca del río Totare, Colombia. En: G. Cervantes (Ed.). Memorias del XLII Congreso Nacional de Ciencias Biológicas. Departamento de Publicaciones, Universidad Simón Bolívar, Barranquilla. p. 35.

Vásquez J „, R amírez, F. (2008). Aspectos bioecológicos del orden Trichoptera en su estado larval en la cuenca del río Totare, departamento del Tolima. Trabajo de grado (Biólogo). Universidad del Tolima, Facultad de Ciencias Básicas, Departamento de Biología, Ibagué.

Vásquez J , Ramírez, F., Reinoso, G., Guevara, G. (2008a). Hydroptílidos (Trichoptera) de la cuenca del río Totare, Tolima-Colombia. En: E. Peña, J. Cantera, M. Zúñiga, S. Duque, E. Londoño, L. Aguirre, B. Albán, P. Bedoya \& D. Santofimio (Eds.). Resúmenes del VIII Seminario Colombiano de Limnología y I Reunión Internacional de Cuencas Bajas y Zonas Estuarinas. Universidad Autónoma de Occidente, Santiago de Cali.

Vásquez J „, Ramírez, F., Reinoso, G., Guevara, G. (2008b). Estudio de los tricópteros en su estado larval en la cuenca del río Totare, Tolima, Colombia. En: P. Landázuri (Ed.). 
Memorias XLIII Congreso Nacional de Ciencias Biológicas. Armenia, Quindío. p. 31.

Vásquez-R amos, J. M., R amírez-Díaz, F., R einoso, G . (2010). Distribución espacial y temporal de los tricópteros inmaduros en la cuenca del río Totare (Tolima-Colombia). Caldasia, 32 (1): 129-148.

Vásquez, J . (2012). Composición y estructura de tricópteros en cuencas con bosque seco tropical del departamento del Tolima. Universidad del Tolima. Trabajo de grado (Magister en Ciencias Biológicas). Universidad del Tolima, Facultad de Ciencias Básicas, Departamento de Biología, Ibagué.
Vergara R., Góngora, F., Prieto, M., Galeano, P. (1994). Inventario de la entomofauna acuática de la quebrada Padilla, fuente del acueducto de Honda (Tolima). Revista Colombiana de Entomología. 20 (2): 115-123.

Wallace J B. 1971 A progress report on the North American Macronema larvae: Their retreats, food and feeding nets (Trichoptera: Hydropsychidae). Proceedings of the First International Symposium on Trichoptera. p. 145-146.

Wiggins G.B. (1996). Larvae of the North American Caddisfly Genera (Trichoptera), 2nd edition. University of Toronto Press, Toronto, p. 456. 